ARTIGO DE ATUALIZAÇÃO UPDATE ARTICLE

Palavras-chave:

remuneração, assistência à saúde, eficiência organizacional

\section{O modelo de remuneração definindo a forma de cuidar: por que premiar a ineficiência no cuidado ao idoso?}

\author{
The remuneration model defining care: why is \\ inefficiency in elderly care rewarded? \\ Martha Regina de Oliveira', Renato Peixoto Veras², Hésio Albuquerque Cordeiro ${ }^{3}$ \\ DOI: 10.21115/JBES.v10.n2.p198-202
}

\section{RESUMO}

O modelo hegemônico de remuneração dos serviços de saúde em muitos países, tanto em sistemas públicos quanto naqueles orientados ao mercado de planos privados de saúde, ainda é o de fee-for-service. Este se caracteriza, essencialmente, pelo estímulo à competição por usuários e remuneração por quantidade de serviços produzidos (volume). Não basta mudar o modelo de remuneração sem alterar o modelo assistencial e vice-versa, pois os dois são interdependentes. O importante na escolha de um modelo diferenciado de remuneração é que seja adequado ao tipo de assistência executado e ao objetivo que se deseja atingir. Ao longo de anos de aplicação de determinado modelo assistencial associado a um modelo de remuneração, todo um sistema de saúde fica moldado e programado para um fim. Essa é a principal discussão a ser feita atualmente. Alguns dos problemas do sistema de saúde brasileiro, em especial o suplementar, e que afetam primordialmente o idoso são consequência do modelo adotado há décadas. Para dar conta dessa nova e urgente demanda da sociedade, modelos alternativos de remuneração devem ser implementados para romper o círculo vicioso de sucessão de consultas fragmentadas e descontextualizadas da realidade social e de saúde da pessoa idosa, além da produção de procedimentos desconectados do desfecho esperado.

\begin{abstract}
The hegemonic remuneration model in health services in many countries, both in public and private systems is still fee-for-service. This is characterized, essentially, by the stimulus to the competition for doing procedures and remuneration by quantity of services produced (volume). It is not enough to change the remuneration model without changing the care model, as both are interdependent. What is important in choosing a differentiated remuneration model is if it is appropriate to the type of assistance performed and the objective to be achieved. Throughout years of applying a particular care model associated to a compensation model, a whole health system is shaped and programmed to deliver this result. This is the main discussion to be made nowadays. Some of the problems that we have in our health system, especially the supplementary one, and that primarily affect the elderly, are a consequence of the model adopted decades ago. In order to deal with this new and urgent demand from society, alternative remuneration models must be implemented to break with the vicious circle of succession of fragmented health care of the elderly, as well as the production of procedures disconnected from the expected outcome.
\end{abstract}

Recebido em: 16/04/2018. Aprovado para publicação em: 30/07/2018.

1. Médica, doutoranda em Envelhecimento, Faculdade de Ciências Médicas, Universidade do Estado do Rio de Janeiro, Rio de Janeiro, RJ, Brasil.

2. Professor-Associado do Instituto de Medicina Social, Diretor da Universidade Aberta da Terceira Idade, Universidade do Estado do Rio de Janeiro (UnATI/UERJ), Rio de Janeiro, RJ, Brasil.

3. Professor visitante do Instituto de Medicina Social e pesquisador da Universidade Aberta da Terceira Idade, Universidade do Estado do Rio de Janeiro (UnATI/UERJ), Rio de Janeiro, RJ, Brasil.

Instituição onde o trabalho foi realizado: Universidade Aberta da Terceira Idade, Universidade do Estado do Rio de Janeiro (UnATI/ UERJ), Rio de Janeiro, RJ, Brasil.

Fontes de financiamento: $\mathrm{o}$ estudo não recebeu fontes externas de financiamento.

Conflito de interesses: este artigo é parte integrante de tese de doutorado.

Autora correspondente: Martha Regina de Oliveira. Rua São Francisco Xavier, 524 - $10^{\circ}$ andar - Bloco F - Maracanã - Rio de Janeiro, RJ, Brasil. CEP 20550-110. Telefones: (21) 2334-0053/2334-0131.E-mail: martha@scaff.med.br 


\section{Introdução}

Há mais de uma década, em um Relatório que se tornou referência mundial intitulado Crossing the quality chasm: a new health system for the 21st century, o Instituto de Medicina dos Estados Unidos declarou a necessidade de alinhamento da remuneração dos serviços de atenção à saúde a mecanismos de indução e medidas de mensuração da qualidade (Institute of Medicine, 2001). O modelo hegemônico de remuneração dos serviços de saúde em muitos países, tanto em sistemas públicos de saúde quanto naqueles orientados ao mercado de planos privados de saúde, ainda é o de fee-for-service (FFS). Este se caracteriza essencialmente pelo estímulo à competição por usuários e remuneração por quantidade (volume) de serviços produzidos (ANS, 2016).

Não basta mudar o modelo de remuneração sem modificar também o modelo assistencial e vice-versa. Os dois são interdependentes, caminham juntos e um reforça e é reforçado pelo outro ao longo dos anos. Além disso, qualquer modelo de remuneração que se adote precisa ter a modelagem baseada num modelo ganha-ganha, segundo o qual todos precisam ser beneficiados: pacientes, profissionais de saúde, prestadores de saúde e operadoras de planos de saúde.

O prolongado processo de transição epidemiológica no Brasil - quase simultâneo ao processo de transição demográfica - coloca para o país um dos seus maiores enfrentamentos futuros em termos de política pública, qual seja, responder às necessidades de atenção à saúde impostas pelas doenças crônicas não transmissíveis, tendo como pano de fundo a atual estrutura de serviços e a mesma dinâmica do modelo assistencial vigente, que em grande parte permanece fragmentária e desarticulada. Em resumo, caso não haja uma mudança radical na atual conformação do modelo de atenção e na prestação dos serviços de saúde, as doenças crônicas não transmissíveis - aliadas ao processo de envelhecimento populacional em curso - poderão obstruir o desenvolvimento econômico das nações, por impactar diretamente a capacidade produtiva, laboral e social das pessoas. Até 2050, o país terá aumento da participação de idosos na população da ordem de três vezes em relação ao cenário atual.

Quando se comparam as projeções de crescimento da população idosa com 80 anos e mais nos principais países da Organização de Cooperação e de Desenvolvimento Econômico (OCDE) - organização internacional composta de 37 países- e no Brasil, percebe-se que nas próximas décadas o país envelhecerá de forma bem mais acelerada do que ocorreu na maioria desses países.

\section{Metodologia}

Para a descrição dos modelos de remuneração, suas aplicações e resultados, foi realizada uma vasta revisão bibliográfi- ca nas bases SciELO e PubMed (modelos de remuneração). Depois disso, foi realizada uma reflexão sobre o modelo brasileiro, suas consequências e a importância dos modelos de remuneração para o redesenho de um sistema de saúde.

\section{Os modelos de remuneração}

Desde as reformas da década de 1990, países como Inglaterra, França, Alemanha, Austrália e Estados Unidos têm experimentado a transição do modelo FFS único para modelos mistos de remuneração que envolvem até o pagamento por desempenho de prestadores de serviços de saúde, conhecido como pay for performance (P4P) (Petersen et al., 2006; Benzer et al., 2014; Collier, 2012; Kecmanovic \& Hall, 2015; Khullar et al., 2015; Lester et al., 2013). No Brasil, outras formas de remuneração representam apenas $5 \%$ do que é praticado pelo mercado de saúde suplementar e algumas experiências, no sistema público, de pagamento por orçamento global e por capitação foram implementadas nos últimos anos.

A literatura sobre o tema das formas de pagamento de prestadores de serviços de saúde apresenta distintos modelos de remuneração. Vale relatar experiências referentes aos bundled payments for care improvement, ao capitation, aos pagamentos globais e às accountable care organizations. Destaca-se que todas as abordagens foram, em um ou outro contexto, bem-sucedidas e amplamente adotadas em diferentes países. As características de cada método fazem com que sejam aplicáveis a todos os perfis da saúde ou a um âmbito particular.

O importante na escolha de um modelo diferenciado de remuneração é que seja adequado ao perfil do tipo de assistência executado e do objetivo que se deseja atingir. Deve-se pensar nos modelos como uma régua de risco, estando de um lado o fee-for-service, com o "risco" todo no pagador, e no outro extremo o capitation, com todo o "risco" no prestador. No meio estariam as formas de compartilhamento desse risco entre pagadores e prestadores de serviço.

Ao longo de anos de aplicação de um modelo assistencial associado a um modelo de remuneração, todo um sistema de saúde fica moldado e programado para determinado fim. Essa é a principal discussão a ser feita hoje. Alguns dos problemas presentes no sistema de saúde brasileiro, em especial o suplementar, e que afetam primordialmente o idoso, por acessar mais o sistema de saúde, são consequência do modelo adotado ao longo de décadas.

Como a essência do FFS é o pagamento por produção de procedimentos individuais, uma das principais desvantagens desse modelo é o estímulo à sobreutilização de serviços intermediários, principalmente os que proporcionam margens de lucro mais elevadas e, portanto, afetam de forma negativa a qualidade da atenção à saúde. Hoje o FFS é o 
modelo mais associado na literatura ao aumento desnecessário no custo da assistência médica.

Vale destacar que o FFS não desaparecerá. Mesmo nos modelos nos quais se optou por um tipo diferente de remuneração, o FFS permaneceu isoladamente em alguns casos específicos ou em associação a outros métodos. O que se discute aqui é a necessidade de um modelo misto, que reforce e incentive características do modelo assistencial almejado, com qualidade, desfecho e satisfação do paciente (Tabela 1).

\section{Mas o que isso tem a ver com o idoso? E por que mudar?}

Em tese, não há que se falar em modelos de remuneração de serviços voltados a populações específicas como a dos idosos. Autores especializados no tema postulam que os modelos de remuneração são orientados ao contexto de sua implementação, quer seja o hospital, a atenção especializada, a atenção primária, quer um conjunto de profissionais de saúde.

Tabela 1. Tipologia e características das formas de remuneração da prestação de serviços de saúde

\begin{tabular}{|c|c|c|c|c|}
\hline Forma de remuneração & Sinonímias & Alocação do recurso & Foco & $\begin{array}{l}\text { Tipo de informação } \\
\text { coletada }\end{array}$ \\
\hline \multirow[b]{2}{*}{ Fee-for-service } & $\begin{array}{l}\text { Por unidade de serviço } \\
\text { Pagamento por ato } \\
\text { médico (conta aberta) }\end{array}$ & Ex-Post & Faturamento & $\begin{array}{l}\text { Custo unitário das } \\
\text { unidades de serviço }\end{array}$ \\
\hline & $\begin{array}{l}\text { Preço fixo (pacotes } \\
\text { de procedimentos e } \\
\text { diárias hospitalares) }\end{array}$ & Ex-Ante & Custos & $\begin{array}{c}\text { Custo unitário das } \\
\text { unidades de serviços } \\
+ \\
\text { Protocolos clínicos/ } \\
\text { cirúrgicos }\end{array}$ \\
\hline Assalariamento & Remuneração por tempo & Ex-Post & Custos & $\begin{array}{c}\text { Custos de transação } \\
\text { e administrativos }\end{array}$ \\
\hline Orçamento global & $\begin{array}{l}\text { Transferência } \\
\text { orçamentária }\end{array}$ & Ex-Ante & $\begin{array}{c}\text { Desempenho do } \\
\text { prestador conforme } \\
\text { metas contratualizadas }\end{array}$ & $\begin{array}{l}\text { Custos + metas por } \\
\text { desempenho }\end{array}$ \\
\hline $\begin{array}{l}\text { Grupos de diagnósticos } \\
\text { homogêneos (diagnose } \\
\text { related groups - DRG) }\end{array}$ & $\begin{array}{l}\text { Remuneração por } \\
\text { caso (Case-Mix) }\end{array}$ & Ex-Post & Case-Mix & $\begin{array}{l}\text { Custo por grupos } \\
\text { de diagnóstico } \\
\text { ajustados por risco }\end{array}$ \\
\hline $\begin{array}{l}\text { Prepaid/capitation } \\
\text { (pagamento por usuário) }\end{array}$ & Partial Capitation* & Ex-Ante & Pessoas/Risco & $\begin{array}{c}\text { Custo unitário das } \\
\text { unidades de serviços } \\
+ \\
\text { Protocolos clínicos/ } \\
\text { cirúrgicos } \\
+ \\
\text { Incidência dos } \\
\text { procedimentos } \\
\text { hospitalares }\end{array}$ \\
\hline $\begin{array}{l}\text { Bundled payments for } \\
\text { care improvement }\end{array}$ & $\begin{array}{l}\text { Bundled payments for } \\
\text { care improvement (BPCl); } \\
\text { bundled payments for } \\
\text { acute care (inpatient only); } \\
\text { bundled payments for } \\
\text { episodes of care (agudos ou } \\
\text { crônicos); condition-specific } \\
\text { budget (medical home) }\end{array}$ & $\begin{array}{l}\text { Maiora Ex-Post } \\
\quad(E \cup A)\end{array}$ & $\begin{array}{c}\text { Caso } \\
\text { Episódio clínico } \\
\text { Condição de saúde }\end{array}$ & $\begin{array}{c}\text { Custo + medidas de } \\
\text { qualidade + coordenação } \\
\text { do cuidado }\end{array}$ \\
\hline Shared savings programs & $\begin{array}{l}\text { Remuneração por } \\
\text { economias geradas }\end{array}$ & Ex-Post & $\begin{array}{l}\text { Relaciona pagamento- } \\
\text {-compartilhamento de } \\
\text { risco a qualidade do } \\
\text { cuidado prestado }\end{array}$ & $\begin{array}{c}\text { Custo + medidas de } \\
\text { qualidade + coordenação } \\
\text { do cuidado }\end{array}$ \\
\hline $\begin{array}{l}\text { Pagamento por } \\
\text { perfomance }\end{array}$ & Pay-for-Performance (P4P) & Ex-Post & $\begin{array}{c}\text { Desempenho-metas } \\
\text { do prestador qualidade } \\
\text { do cuidado }\end{array}$ & $\begin{array}{c}\text { Custo }+ \text { medidas de } \\
\text { qualidade/desempenho } \\
\text { do prestador }\end{array}$ \\
\hline
\end{tabular}

* A Lei define que, no pagamento por Capitação Parcial, a ACO estaria sob risco financeiro para alguns itens e serviços cobertos pelas partes A e B do Medicare, por exemplo, para todas as consultas médicas ou todos os itens e serviços cobertos pela parte B.

Fonte: adaptada de ANS - Projeto Idoso Bem Cuidado - Idosos na saúde suplementar: uma urgência para a saúde da sociedade e sustentabilidade do setor, 2016. 
Algumas características, no entanto, ressaltadas quando da construção de um modelo de cuidado, podem servir de parâmetro para que se possa refletir sobre a adequação desta ou daquela forma de remuneração da atenção ao idoso. A primeira delas é a reorganização dos arranjos assistenciais para prestação do cuidado às pessoas idosas. A principal diretriz dessas mudanças nos arranjos assistenciais, que também envolvem a remuneração dos serviços, deve ter em conta que a população idosa, mesmo saudável, possui algum grau de fragilidade. Assim, é necessário identificar esse risco de fragilização precocemente para que medidas preventivas possam ser estabelecidas, mantendo esse idoso, pelo maior tempo possível, numa condição de autonomia e independência. Para tanto, instrumentos de rastreio dessa fragilidade, que possuam caráter multidimensional e não somente clínico, devem ser adotados para a realidade brasileira. A complexidade desse modelo mais integrativo e contínuo do cuidado em saúde, típico de populações mais sujeitas a doenças crônicas, requer profundas mudanças nos modelos de pagamento dos prestadores de saúde para que possam de fato acontecer atreladas às mudanças na lógica do modelo assistencial.

Outro aspecto a ser ressaltado é a necessidade de coordenação do cuidado, no sentido do estabelecimento de mecanismos de referência e contrarreferência, para que os dispositivos que compõem a rede assistencial estejam em sintonia e facilitem o itinerário do idoso por meio de suas diferentes estruturas [atenção primária, unidades de acolhimento (retaguarda), emergências, hospitais e serviços de cuidados prolongados e cuidados paliativos]. Esse novo desenho requer a adequação das estruturas da rede e dos profissionais que a integram, capacitando-os a reconhecer essa nova lógica de cuidado e construindo um sistema de informação em saúde que estabeleça os caminhos necessários para um cuidado mais integrativo.

Para dar conta dessa nova e urgente demanda da sociedade, modelos alternativos de remuneração devem ser implementados para romper o círculo vicioso de sucessão de consultas fragmentadas e descontextualizadas da realidade social e de saúde da pessoa idosa, além da produção de procedimentos desconectados do desfecho esperado, o que, para o idoso, pode trazer consequências ainda mais importantes do que para um jovem, ou seja, um novo paradigma no modelo assistencial.

Para entender a importância de um modelo de remuneração no reforço das estratégias de um modelo assistencial, será pontuada, a seguir, de que maneira a utilização do FFS de forma isolada durante décadas no Brasil trouxe consequências. Registra-se, ainda, onde é possível atuar para mudar ambos os modelos (assistencial e de remuneração), de modo a atingir os melhores resultados no sistema.

\section{Na formação de profissionais da saúde}

O modelo de remuneração vigente privilegia, como já relatado, a possibilidade de produção de procedimentos e o volume de produção. Assim sendo, ao longo dos anos, as especialidades que realizam procedimentos para além da consulta foram sendo "privilegiadas" em termos de remuneração. Hoje existe uma importante distorção na formação tanto de profissionais que têm especialidades com essas características quanto dos que não as têm. O melhor exemplo disso é a relação candidato/vaga de especializações e residências como Oftalmologia, Dermatologia e Cirurgias versus Clínica Médica, Geriatria ou Pediatria.

\section{No equipamento em saúde disponível: paliativo e transição versus unidade de terapia intensiva (UTI)}

Da mesma forma que a formação dos profissionais de saúde, a estrutura de equipamentos no sistema de saúde também foi desenhada através do tempo pelo que é ou não mais bem remunerado. Assim, encontram-se com facilidade aberturas de leitos de UTI, que representam tecnologia em saúde e que ao longo do tempo foram privilegiadas no modelo, enquanto praticamente há disponibilidade de leitos de cuidado paliativo, que são aqueles com muito menos tecnologia agregada e historicamente "desvalorizados" pelo atual sistema.

Ora, quanto maior o envelhecimento da população, maior a necessidade desse tipo de leito. Contudo, como incentivar sua existência se o modelo de remuneração pende totalmente para mais tecnologia?

\section{A opção pela "boa morte"}

Totalmente atrelada à discussão anterior, como é possível discutir o conceito de boa morte, principalmente na saúde suplementar brasileira, se não há instrumentos para isso? Teria hoje um paciente a possibilidade de ter uma morte diferente da morte invasiva e repleta de procedimentos? Isso tem influência direta da disponibilidade de serviços, mas também de conceitos culturais também moldados e influenciados durante anos pelo modelo de remuneração e assistencial vigentes.

A cultura dos pacientes sendo moldada pela lógica do sistema (consumo, imediatismo e utilização de tecnologia).

A mesma lógica que permeou a organização do sistema de saúde e seus profissionais também foi construindo a cultura da sociedade sobre o que seria um bom tratamento ou um bom sistema de saúde. Assim, a sociedade em geral também passou a valorizar consumo e tecnologia, pois foi isso que o próprio sistema de saúde lhe ensinou a desejar e a conhecer como valor nos seus cuidados. Também é necessário promover debates sobre educação em saúde, assim como valorizar o que é importante dentro do sistema. 


\section{Especialistas e hipermedicação}

Como uma das consequências da desorganização do sistema, da falta de integração de informações e da busca por especialistas de forma desordenada, a hipermedicação é um dos maiores problemas no cuidado ao idoso.

Excesso de exames e procedimentos e o não "caminhar" no tratamento

Como consequência dos modelos atuais (assistencial e de remuneração), ocorre a sobreutilização de procedimentos. Além da própria sobreutilização, esse processo muitas vezes atrasa o caminhar do paciente em seu plano terapêutico, uma vez que repete várias vezes a mesma etapa, dificultando o acesso à próxima.

\section{Urgência e emergência versus cuidados primários e prolongados}

Da mesma forma como em outros pontos aqui destacados a cultura do imediatismo e da produção de procedimentos -, a porta aberta da emergência faz mais sentido no atual sistema de saúde do que a organização e a continuidade de cuidado da atenção primária.

\section{Conclusão}

Os modelos assistenciais e de remuneração devem caminhar juntos. Ao mudar um, é preciso atentar para o outro, para que as ações se potencializem.

Alguns pontos merecem destaque na conclusão deste texto: não importa que modelo de remuneração seja escoIhido, desde que seja pensado para reforçar o resultado assistencial que se deseja obter. Também não existe uma fórmula mágica para construir um modelo de remuneração, mas modelos que usam tipos mistos (exemplo: FFS + performance) e são aplicados de forma gradual; observando-se seus efeitos na área assistencial, eles apresentam melhores resultados.
Outro ponto importante a destacar, como cita o Institute for Healthcare Improvement ao descrever a situação dos sistemas de saúde: "Todo sistema é perfeitamente desenhado para atingir os resultados que atinge" (Berwick, 2011a, 2011b). É necessário que todos os atores de um sistema de saúde realmente queiram mudar o sistema. Mais uma vez, é reforçada a importância do sistema ganha-ganha.

Não é fácil mudar um sistema de saúde, mas vários outros países já provaram ser possível. A necessidade é real e urgente.

\section{Referências bibliográficas}

Agência Nacional de Saúde Suplementar. Projeto Idoso Bem Cuidado: "Idosos na saúde suplementar: uma urgência para a saúde da sociedade e sustentabilidade do setor". Rio de Janeiro: ANS, 2016.

Benzer JK, Young GJ, Burgess Jr JF, Baker E, Mohr DC, Charns MP, et al. Sustainability of quality improvement following removal of pay-forperformance incentives. J Gen Intern Med. 2014;29(1):127-32.

Berwick DM. Launching accountable care organizations - The proposed rule for the Medicare Shared Savings Program. N Engl J Med. 2011a;364(16): e32.

Berwick DM. Making good on ACOs' promise - The final rule for the Medicare shared savings program. N Engl J Med. 2011b;365(19):1753-6.

Collier R. Professionalism: how payment models affect physician behaviour. Can Med Assoc J. 2012;184(12):E645-E646.

Institute of Medicine. Committee on Quality of Health Care in America. Crossing the quality chasm: a new health system for the 21st century. Washington, DC: National Academy Press, 2001.

Kecmanovic M, Hall JP. The use of financial incentives in Australian general practice. Med J Aust. 2015;202(9):488-91.

Khullar D, Chokshi DA, Kocher R, Reddy A, Basu K, Conway PH, et al. Behavioral economics and physician compensation - Promise and challenges. N Engl J Med. 2015;372(24):2281-3.

Lester H, Matharu T, Mohammed MA, Lester D, Foskett-Tharby R. Implementation of pay for performance in primary care: a qualitative study 8 years after introduction. Br J Gen Pract. 2013;63(611):e408-e415.

Petersen LA, LeChauncy DW, Urech T, Daw C, Sookanan S. Does pay-forperformance improve the quality of health care? Ann Intern Med. 2006 145(4):265-72. 